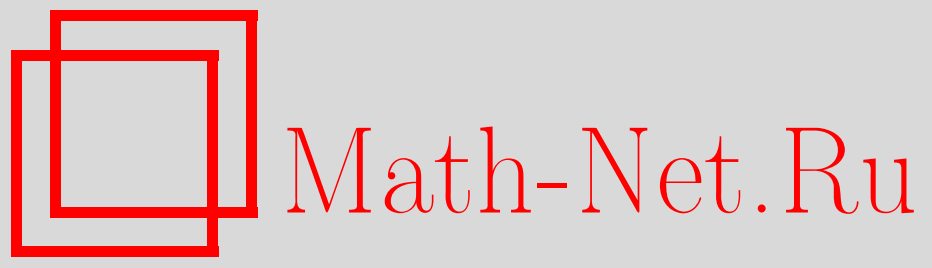

W. Hong, H. Wang, Intrinsic branching structure within random walk on Z, Теория вероятн. и ее примен., 2013, том 58, выпуск 4, 730-751

DOI: https://doi.org/10.4213/tvp4538

Использование Общероссийского математического портала Math-Net.Ru подразумевает, что вы прочитали и согласны с пользовательским соглашением http://www . mathnet.ru/rus/agreement

Параметры загрузки:

IP: 54.84 .234 .179

26 апреля 2023 г., $17: 35: 41$

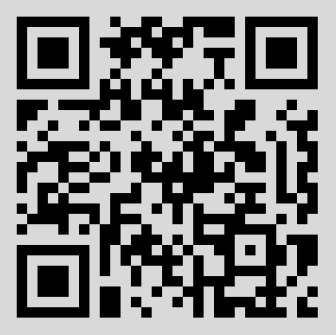




\title{
INTRINSIC BRANCHING STRUCTURE WITHIN RANDOM WALK ON $\mathbf{Z}^{1)}$
}

\begin{abstract}
В работе описан ветвящийся процесс, вкладываемый в неоднородное случайное блуждание, имеющее ограниченные приращения. В терминах этого неоднородного по времени многотипного ветвящегося процесса можно выразить лестничный момент $T_{1}$, момент первого достижения множества $[1, \infty)$ случайным блужданием, стартующим из 0. В качестве примера использования упомянутого ветвящегося процесса мы доказываем закон больших чисел для случайного блуждания, имеющего ограниченные скачки и эволюционирующего в случайной среде, а также находим в явном виде инвариантную плотность для цепи Маркова, порожденной «средой, наблюдаемой частицами». Инвариантная плотность и предельная скорость цепи может быть найдена в явном виде в терминах этой среды.
\end{abstract}

Ключевье слова и фразы: случайное блуждание, ветвящийся процесс, случайная среда, инвариантная плотность.

\section{Introduction.}

1.1. Background and motivation. We study random walk $\left\{X_{n}\right\}$ with bounded jumps in this paper. Precisely, for $L, R \geqslant 1$, let $\Lambda=$ $\{-L, \ldots, R\} /\{0\}$. At position $x$ the walk jumps with probability $\omega_{x}(l)$ to $x+l$ for $l \in \Lambda$. Of course, $\sum_{l \in \Lambda} \omega_{x}(l)=1$ and $\omega_{x}(l) \geqslant 0, l \in \Lambda$. We call $\left\{X_{n}\right\}$ the (L-R) random walk hereafter.

Let $T_{1}$ be the time the walk hits the positive half line $[1, \infty)$ for the first time. It is a fundamental task to characterize $T_{1}$, which plays an important roles in studying the (L-R) random walk, for example, the recurrence vs transience, law of large numbers (LLN hereafter), central limits theorem and large deviations principle et al.

For (1-1) random walk with nonhomogeneous transition probabilities, that is, $\omega_{x}$ depends on $x$, it has been revealed in [5] that $T_{1}$ could be expressed

${ }^{*}$ School of Mathematical Sciences \& Key Laboratory of Mathematics and Complex Systems, Beijing Normal University, Beijing 100875, P.R.China; e-mail: wmhong@bnu.edu.cn

** Department of Mathematics, Anhui Normal University, Wuhu 241003, P.R.China; e-mail: hmking@mail.ahnu.edu.cn

1) This project is partially supported by the National Nature Science Foundation of China (grants № № 11131003, 11226199) and 985 project. 
in terms of a nonhomogeneous Galton-Watson branching process. This fact enables them to prove a nice stable limit theorem for nearest neighbor Random Walk in Random Environment (RWRE hereafter). If $\max \{L, R\}>1$, that is, for the nonnearest neighbor random walk, things get very different.

For (L-1) random walk, Hong and Wang in [3] revealed its branching structure, that is, $T_{1}$ could be expressed by a multitype ( $L$-type) branching process. Using the branching structure, they also proved a stable limit theorem, partially generalizing the results, for (1-1) RWRE, of [5] to (L-1) RWRE which is supposed to be transient to the right.

Hong and Zhang (see [4]) considered the (1-R) setting. The authors decomposed the random walk path to reveal that $T_{1}$ for (1-R) random walk could be expressed by a nonhomogeneous $(1+2+\cdots+R)$-type branching process and by this fact they also proved an LLN for (1-R) RWRE by a method known as «the environment viewed from particles».

One should note that, both the above mentioned [3] and [4] treat the case $\min \{L, R\}=1$. The walk is requested to be nearest neighbor at least in one side. The main purpose of this paper is to consider the general situation: $L, R \geqslant 1$. We restrict ourselves to $L=R=2$ to explain the idea. We reveal the intrinsic branching structure within the (L-R) random walk and give some applications

1.2. The model. Fix $L, R \geqslant 1$. Set $\Lambda=\{-L, \ldots, R\} /\{0\}$. For $i \in \mathbf{Z}$, let $\omega_{i}=\left(\omega_{i}(l)\right)_{l \in \Lambda}$ be a probability measure on $i+\Lambda$, that is, $\sum_{l \in \Lambda} \omega_{i}(l)=1$ and $\omega_{i}(l) \geqslant 0$ for all $l \in \Lambda$. Set $\omega=\left\{\omega_{i}\right\}_{i \in \mathbf{Z}}$, which will serve as the transition probabilities of the random walk. Let $\left\{X_{n}\right\}_{n \geqslant 0}$ be a Markov chain with initial value $X_{0}=x$ and transition probabilities

$$
P_{\omega}\left(X_{n+1}=i+j \mid X_{n}=i\right)=\omega_{i}(j), \quad j \in \Lambda \text {. }
$$

We call $\left\{X_{n}\right\}$ the (L-R) random walk with nonhomogeneous transition probabilities. Throughout, we use $P_{\omega}^{x}$ to denote the law induced by random walk $\left\{X_{n}\right\}$ and $E_{\omega}^{x}$ the corresponding integration operator.

In the remainder of the paper, in order to avoid the heavy notation we consider the case $L=2, R=2$, that is, the (2-2) random walk. The idea for treating the general (L-R) random walk is basically the same as (2-2) setting. Also except otherwise stated, we always assume the random walk starts from 0 .

For (2-2) random walk $\left\{X_{n}\right\}$, set $T_{0}=0$ and define recursively

$$
T_{k}=\inf \left[n \geqslant 0: X_{n}>X_{T_{k-1}}\right]
$$

for $k \geqslant 1$. We call the stopping times $T_{k}, k \geqslant 1$, the ladder times of the random walk.

Especially one sees by the definition that

$$
T_{1}=\inf \left[n \geqslant 0: X_{n}>0\right]
$$


is the hitting time of $[1, \infty)$ by the walk, and $X_{T_{1}}$ is also a random variable with two possible values $X_{T_{1}}=1$ or $X_{T_{1}}=2$. This is the reason why we call $T_{k}, k \geqslant 1$, the ladder times. The distribution of $X_{T_{1}}$, the exit probabilities of the walk from $(-\infty, 0]$, could be calculate by some standard procedure.

1.3. The main results. Our purpose is to count the total steps of the walk before $T_{1}$. If $T_{1}=\infty$, we need do nothing. So we assume throughout $T_{1}<\infty$ which follows immediately if one assumes that $\lim \sup _{n \rightarrow \infty} X_{n}=\infty$. We use several lines to give an equivalent condition to characterize $\lim \sup _{n \rightarrow \infty} X_{n}=\infty$.

Indeed, fix $a<b$. Let $\partial^{+}[a, b]=\{b, b+1\}$ and $\partial^{-}[a, b]=\{a, a-1\}$ be the positive and negative boundaries of $[a, b]$, respectively. For $k \in(a, b)$, $\zeta \in \partial^{+}[a, b] \cup \partial^{-}[a, b]$, define

$$
\left.\mathbf{P}_{k}(a, b, \zeta)=P_{\omega}^{k} \text { (the walk exits the interval }[a+1, b-1] \text { at } \zeta\right) .
$$

For simplicity, we write $\mathbf{P}_{k}(a, b, \zeta)$ as $\mathbf{P}_{k}(\zeta)$ temporarily. From the Markov property it follows that

$$
\mathbf{P}_{k}(\zeta)=\omega_{k}(2) \mathbf{P}_{k+2}(\zeta)+\omega_{k}(1) \mathbf{P}_{k+1}(\zeta)+\omega_{k}(-1) \mathbf{P}_{k-1}(\zeta)+\omega_{k}(-2) \mathbf{P}_{k-2}(\zeta),
$$

which leads to the following matrix form:

$$
V_{k}(\zeta)=M_{k} V_{k+1}(\zeta)
$$

where

$$
\begin{gathered}
V_{k}(\zeta):=\left(\begin{array}{c}
\left(\mathbf{P}_{k-1}-\mathbf{P}_{k-2}\right)(\zeta) \\
\left(\mathbf{P}_{k}-\mathbf{P}_{k-1}\right)(\zeta) \\
\left(\mathbf{P}_{k+1}-\mathbf{P}_{k}\right)(\zeta)
\end{array}\right), \\
M_{k}=\left(\begin{array}{ccc}
-\frac{\omega_{k}(-1)+\omega_{k}(-2)}{\omega_{k}(-2)} & \frac{\omega_{k}(1)+\omega_{k}(2)}{\omega_{k}(-2)} & \frac{\omega_{k}(2)}{\omega_{k}(-2)} \\
1 & 0 & 0 \\
0 & 1 & 0
\end{array}\right) .
\end{gathered}
$$

Then $\mathbf{P}_{k}(a, b, b)$ and $\mathbf{P}_{k}(a, b, b+1)$ follows from some standard procedure. They could be expressed in terms of $\left\{M_{i}\right\}_{i \in \mathbf{Z}}$.

One notes that $\lim \sup _{n \rightarrow \infty} X_{n}=\infty$ if and only if $\mathbf{P}_{0}(-\infty, b, b)+$ $\mathbf{P}_{0}(-\infty, b, b+1)=1$ for all $b>0$. Therefore, from here on, we always assume that $\lim \sup _{n \rightarrow \infty} X_{n}=\infty$.

In order to count exactly all steps of the walk before $T_{1}$, we define three types of excursions.

Definition 1.1. (a) We call excursions of the form $\left\{X_{k}=\right.$ $\left.i, X_{k+1}=i-1, X_{k+2} \leqslant i-1, \ldots, X_{k+l} \leqslant i-1, X_{k+l+1} \geqslant i\right\}$ type- $\mathscr{A}$ excursions at $i$. Corresponding to the possible three kinds of last step of type- $\mathscr{A}$ excursions at $i$, say, $\{i-1 \rightarrow i\},\{i-2 \rightarrow i\}$, and $\{i-1 \rightarrow i+1\}$, we classify type- $\mathscr{A}$ excursions at $i$ into three sub-types $\mathscr{A}_{i, 1}, \mathscr{A}_{i, 2}$, and $\mathscr{A}_{i, 3}$. 
(b) We call excursions of the form $\left\{X_{k}=i, X_{k+1}=i-2, X_{k+2} \leqslant\right.$ $\left.i-1, \ldots, X_{k+l} \leqslant i-1, X_{k+l+1} \geqslant i\right\}$ type- $\mathscr{B}$ excursions at $i$. Corresponding to the possible three kinds of last step of type- $\mathscr{B}$ excursions at $i$, say, $\{i-1 \rightarrow i\}$, $\{i-2 \rightarrow i\}$, and $\{i-1 \rightarrow i+1\}$, we classify type- $\mathscr{B}$ excursions at $i$ into three subtypes $\mathscr{B}_{i, 1}, \mathscr{B}_{i, 2}$, and $\mathscr{B}_{i, 3}$.

(c) We call excursions of the form $\left\{X_{k}=i+1, X_{k+1}=i-1, X_{k+2} \leqslant\right.$ $\left.i-1, \ldots, X_{k+l} \leqslant i-1, X_{k+l+1} \geqslant i\right\}$ type- $\mathscr{C}$ excursions at $i$. Corresponding to the possible three kinds of last step of type- $\mathscr{C}$ excursions at $i$, say, $\{i-1 \rightarrow i\}$, $\{i-2 \rightarrow i\}$ and $\{i-1 \rightarrow i+1\}$, we classify type- $\mathscr{C}$ excursions at $i$ into three sub-types $\mathscr{C}_{i, 1}, \mathscr{C}_{i, 2}$, and $\mathscr{C}_{i, 3}$.

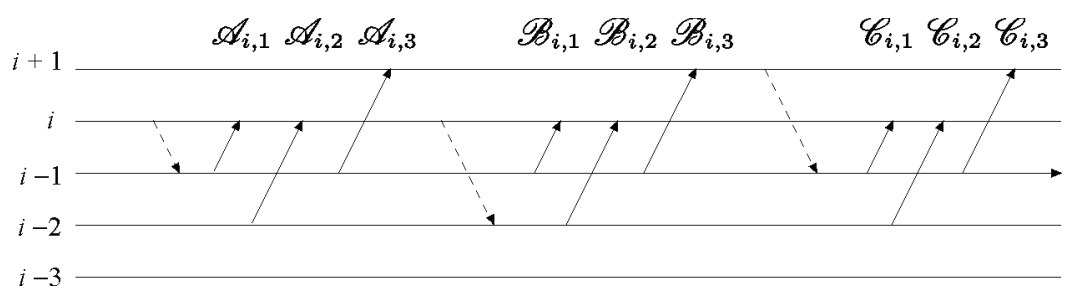

Fig. 1. The figure illustrates type $\mathscr{A}, \mathscr{B}$, and $\mathscr{C}$ excursions at $i$. We draw only the first step and the last step, omitting all things between these two steps. Between these two steps, the walk walks below $i-1$.

Define $A_{i, j}=\#\left\{\mathscr{A}_{i, j}\right.$ excursions before $\left.T_{1}\right\}, B_{i, j}=\#\left\{\mathscr{B}_{i, j}\right.$ excursions before $\left.T_{1}\right\}, C_{i, j}=\#\left\{\mathscr{C}_{i, j}\right.$ excursions before $\left.T_{1}\right\}$, for $i \leqslant 0$ and $j=1,2,3$, where $\#\{\}$ means the number of the elements in set \{\} .

To count all steps by the walk before $T_{1}$, define

$$
U_{i}=\left(A_{i, 1}, A_{i, 2}, A_{i, 3}, B_{i, 1}, B_{i, 2}, B_{i, 3}, C_{i, 1}, C_{i, 2}, C_{i, 3}\right),
$$

being the total numbers of different excursions at $i$ before time $T_{1}$. Then we have the following fact.

Theorem 1.1. Suppose that $\limsup _{n \rightarrow \infty} X_{n}=\infty$. Then

$$
\begin{aligned}
T_{1} & =1+\sum_{i \leqslant 0} U_{i}(2,2,1,1,1,0,2,2,1)^{\mathrm{T}} \\
E_{\omega}^{0}\left(T_{1}\right) & =1+\sum_{i \leqslant 0} u_{1} Q_{0} \cdots Q_{i}(2,2,1,1,1,0,2,2,1)^{\mathrm{T}},
\end{aligned}
$$

where $Q_{i} \in \mathbf{R}^{9} \times \mathbf{R}^{9}, u_{1} \in \mathbf{R}^{9}$ depending only on $\omega$ are defined in (27), (29) below and $\mathbf{v}^{\mathrm{T}}$ denotes the transpose of vector $\mathbf{v}$.

The proof of Theorem 1.1 will be given in Section 3 .

$\mathrm{R}$ e $\mathrm{m}$ a r k 1.1. Because the (2-2) random walk is nonnearest neighbor, it is hard to give the exact distribution of $T_{1}$ in general. But the process $\left\{U_{i}\right\}_{i \leqslant 0}$ defined in (1) will be proved to be a nonhomogeneous multitype branching process. This fact together with (2) enables us to study $T_{1}$ by the properties of branching processes. 
Firstly, we formulate an ancestor for the branching process. The walk starts from 0 , but before $T_{1}$, there is no jump down from above 1 to 0 . One can imagine that there is a step by the walk from 1 to 0 before it starts from 0 (One can also imagine that this step is from 2 to 0 , but this makes no difference.), that is, set $X_{-1}=1$. Adding this imaginary step, the path $\left\{X_{-1}=1, X_{0}=0, X_{1}, \ldots, X_{T_{1}}\right\}$ forms a type- $\mathscr{A}$ excursion at 1 such that with probability 1 ,

$$
A_{1,1}+A_{1,2}+A_{1,3}=1 .
$$

Then one defines $U_{1}$ as in (1). But since there is no $\mathscr{B}_{1, j}$ and $\mathscr{C}_{1, j}, j=1,2,3$, excursions, $U_{1}$ has only three possible values, that is, $U_{1}=\mathbf{e}_{1}, U_{1}=\mathbf{e}_{2}$, or $U_{1}=\mathbf{e}_{3}$.

We can treat $U_{1}$ as some particle immigrates into the system and call it «immigration» throughout.

Theorem 1.2. Suppose that $\limsup _{n \rightarrow \infty} X_{n}=\infty$. Then $\left\{U_{i}\right\}_{i \leqslant 1}$ is a 9-type nonhomogeneous branching processes with immigration distribution as in (6), (7), and (8) below, and offsprings distributions as in (9)-(12), (14)-(17), (18)-(21), and (22)-(26) below.

The proof of Theorem 1.2 will be given in the long Section 2 .

$\mathrm{R}$ e $\mathrm{m}$ a r k 1.2. (1) When $\omega_{i}(-2)=0$ for all $i \in \mathbf{Z}$, Theorem 1.2 reveals the branching structure of (1-2) random walk and when $\omega_{i}(2)=0$ for all $i \in \mathbf{Z}$, Theorem 1.2 reveals that of (2-1) random walk. Therefore the branching structure in Theorem 1.2 contains both the branching structures of [3] and [4].

(2) The authors found that the branching structure could be simplified. A 6-type branching process is enough to count all steps by the walk before $T_{1}$. However we still use a 9-type branching process, since it is more comprehensible and each of the 9 types of particles corresponds to specific jump of the walk.

At last, as application of the branching structure, we prove an LLN for transient (2-2) random walk in ergodic random environment by a method known as «the environment viewed from particles», which dates back to [6].

To define (2-2) random walk in random environment, let $\Omega$ be the collection of $\omega=\left\{\omega_{i}\right\}_{i \in \mathbf{Z}}$ and let $\mathscr{F}$ be the Borel $\sigma$-algebra on $\Omega$. Define the shift operator on $\Omega$ by

$$
(\theta \omega)_{i}=\omega_{i+1}
$$

Let $\mathbf{P}$ be a probability measure on $(\Omega, \mathscr{F})$ making $(\Omega, \mathscr{F}, \mathbf{P}, \theta)$ an ergodic system. The so-called random environment is a random element $\omega \in \Omega$ chosen according to the probability $\mathbf{P}$.

The (2-2) random walk $\left\{X_{n}\right\}_{n \geqslant 0}$ in random environment $\omega$ is define to be a Markov chain with initial value $X_{0}=x$ and transition probabilities

$$
P_{\omega}\left(X_{n+1}=i+j \mid X_{n}=i\right)=\omega_{i}(j), \quad j \in\{1,2,-1,-2\} .
$$


The measure $P_{\omega}^{x}$ induced by $\left\{X_{n}\right\}$ on $\left(\mathbf{Z}^{\mathbf{N}}, \mathscr{G}\right)$, with $\mathscr{G}$ the Borel $\sigma$ algebra, is called the quenched probability and the probability $P^{x}$ defined on $\left(\mathbf{Z}^{\mathbf{N}}, \mathscr{G}\right)$ by the relation

$$
P^{x}(B)=\int P_{\omega}^{x}(B) \mathbf{P}(d \omega), \quad B \in \mathscr{G},
$$

is called the annealed probability. We show the following LLN.

Theorem 1.3. Suppose that $E^{0}\left(T_{1}\right)<\infty$. Then one has that

$$
\lim _{n \rightarrow \infty} \frac{X_{n}}{n}=V_{\mathbf{P}}
$$

for some $V_{\mathbf{P}}$. Moreover,

$$
V_{\mathbf{P}}=\frac{E_{\mathbf{P}}\left(\Pi(\omega)\left(2 \omega_{0}(-2)+\omega_{0}(-1)+\omega_{0}(1)+2 \omega_{0}(2)\right)\right)}{E_{\mathbf{P}}(D(\omega))},
$$

where $\Pi(\omega)$ and $D(\omega)$ are defined in (37) and (39).

$\mathrm{R}$ e $\mathrm{m}$ a r k 1.3. (1) The role the branching structure plays is to give the invariant density $\Pi(\omega)$ explicitly in terms of the environment $\omega$.

(2) In [1], the author also proved an LLN for (L-R) random walk in random environment, see Theorem 1.10 therein. But Brémont in [1] did not give the specific form of the velocity $V_{\mathbf{P}}$. The branching structure enables us to give the invariant density $\Pi(\omega)$ explicitly, so that we can give the velocity $V_{\mathbf{P}}$ explicitly.

2. Path decomposition. Proofs of Theorem 1.2. Remember that $\limsup _{n \rightarrow \infty} X_{n}=\infty$. That is, the walk $\left\{X_{n}\right\}$ is transient to $\infty$ or recurrent. The notation $\{i \rightarrow j\}$ will be always used to denote a jump (a step) by the walk from $i$ to $j$. Define

$$
T_{1}=\inf \left\{n \geqslant 0: X_{n}>0\right\},
$$

the hitting time of $[1, \infty)$. The purpose of this section is to count all steps by the walk before $T_{1}$.

\subsection{The excursions, corresponding probabilities and immigra-} tion laws. From subsection 1.3 of Section 1, we see that the exit probabilities of the walk from certain interval $(a, b)$ could be expressed in terms of $\omega$. So we always assume that all these exit probabilities are already known in the remainder of the paper. For $k \leqslant i<j$, denote

$$
f_{k}(i, j)=P_{\omega}^{k}(\text { the walk hits }(i, \infty) \text { from below at } j) .
$$

We remark that for (2-2) random walk, in the definition of $f_{k}(i, j)$, the term $j$ only takes values in $\{i+1, i+2\}$. With the notation of subsection 1.3 of Section 1,

$f_{k}(i, i+1)=\mathbf{P}_{k}(-\infty, i+1, i+1) \quad$ and $\quad f_{k}(i, i+2)=\mathbf{P}_{k}(-\infty, i+1, i+2)$. 
Further, we analyze the path of the walk. An excursion will be also called a particle some times. Firstly, we consider type- $\mathscr{A}$ excursion of the walk (see (a) of Definition 1.1).

Note that a type- $\mathscr{A}$ particle at $i$ begins when the walk jumps down from $i$ to $i-1$. After that the walk runs in $(-\infty, i-1]$. At last the walk hits $[i, \infty)$ at some $j$ and the excursion goes to end.

We define some indexes $\alpha_{i, 1}, \alpha_{i, 3}$, and $\alpha_{i, 2}$ correspondingly to $\mathscr{A}_{i, 1}, \mathscr{A}_{i, 3}$, and $\mathscr{A}_{i, 2}$. Let

$$
\begin{aligned}
& \alpha_{i, 1}:=\omega_{i}(-1) \sum_{n, m \geqslant 0} \frac{(n+m) !}{n ! m !}\left[\omega_{i-1}(-1) f_{i-2}(i-2, i-1)\right]^{n} \\
& \times\left[\omega_{i-1}(-2) f_{i-3}(i-2, i-1)\right]^{m} \omega_{i-1}(1), \\
& \alpha_{i, 3}:=\omega_{i}(-1) \sum_{n, m \geqslant 0} \frac{(n+m) !}{n ! m !}\left[\omega_{i-1}(-1) f_{i-2}(i-2, i-1)\right]^{n} \\
& \times\left[\omega_{i-1}(-2) f_{i-3}(i-2, i-1)\right]^{m} \omega_{i-1}(2), \\
& \alpha_{i, 2}:=\omega_{i}(-1)-\alpha_{i, 1}-\alpha_{i, 3} .
\end{aligned}
$$

Since $\alpha_{i, 1}$ differs from $\alpha_{i, 3}$ only in the last term of the product, we explain only the meaning of $\alpha_{i, 1}$. The first term $\omega_{i}(-1)$ is transition probability of the first step of the excursion $\mathscr{A}_{i, 1}$ from $i$ to $i-1$. The last term $\omega_{i-1}(1)$ is transition probability of the last step of the excursion $\mathscr{A}_{i, 1}$ from $i-1$ to $i$. The summation in the center indicates all events occurring between the first step and the last step. In details, before the last step happens, $n$ steps of the form $\{i-1 \rightarrow i-2\}$ and $m$ steps of the form $\{i-1 \rightarrow i-3\}$ occur and the total number of possible combinations of these $m+n$ steps is $C_{n}^{n+m}=(n+m) ! / n ! m !$. The term $\omega_{i-1}(-1) f_{i-2}(i-2, i-1)$ means that, with probability $\omega_{i-1}(-1)$, a step $\{i-1 \rightarrow i-2\}$ occurs, and starting from $i-2$, with probability $f_{i-2}(i-2, i-1)$, it hits $[i-1, \infty)$ at $i-1$. The term $\omega_{i-1}(-2) f_{i-3}(i-2, i-1)$ could be explained analogously.

In fact, $\alpha_{i, 2}$ could be defined similarly as $\alpha_{i, 1}$ and $\alpha_{i, 3}$. But the definition is tedious. We note that after the walk jumps down with probability $\omega_{i}(-1)$ from $i$ to $i-1$, then starting from $i-1$, it hits $[i, \infty)$ with probability 1 . Then the summation of $\alpha_{i, 1}, \alpha_{i, 2}$, and $\alpha_{i, 3}$ should be $\omega_{i}(-1)$. So we define $\alpha_{i, 2}:=\omega_{i}(-1)-\alpha_{i, 1}-\alpha_{i, 3}$. Some easy calculation shows that

$$
\begin{aligned}
\alpha_{i, 1} & =\frac{\omega_{i}(-1) \omega_{i-1}(1)}{1-\omega_{i-1}(-1) f_{i-2}(i-2, i-1)-\omega_{i-1}(-2) f_{i-3}(i-2, i-1)}, \\
\alpha_{i, 3} & =\frac{\omega_{i}(-1) \omega_{i-1}(2)}{1-\omega_{i-1}(-1) f_{i-2}(i-2, i-1)-\omega_{i-1}(-2) f_{i-3}(i-2, i-1)} .
\end{aligned}
$$

Secondly, we consider type- $\mathscr{B}$ excursion (see (b) of Definition 1.1). Note that a type- $\mathscr{B}$ excursion begins when the walk jumps down from $i$ to $i-2$. After that the walk runs in $(-\infty, i-1]$. At last the walk hits $[i, \infty)$ at some $j$ and the excursion goes to end. 
Further, we define some indexes $\beta_{i, 1}, \beta_{i, 2}$, and $\beta_{i, 3}$ correspondingly to $\mathscr{B}_{i, 1}, \mathscr{B}_{i, 3}$, and $\mathscr{B}_{i, 2}$. Let

$$
\begin{aligned}
\beta_{i, 1}:=\omega_{i}(-2) f_{i-2}(i-2, i-1) \sum_{n, m \geqslant 0} & \frac{(n+m) !}{n ! m !}\left[\omega_{i-1}(-1) f_{i-2}(i-2, i-1)\right]^{n} \\
\times & {\left[\omega_{i-1}(-2) f_{i-3}(i-2, i-1)\right]^{m} \omega_{i-1}(1), } \\
\beta_{i, 3}:=\omega_{i}(-2) f_{i-2}(i-2, i-1) \sum_{n, m \geqslant 0} & \frac{(n+m) !}{n ! m !}\left[\omega_{i-1}(-1) f_{i-2}(i-2, i-1)\right]^{n} \\
\times & {\left[\omega_{i-1}(-2) f_{i-3}(i-2, i-1)\right]^{m} \omega_{i-1}(2), }
\end{aligned}
$$

$\beta_{i, 2}:=\omega_{i}(-2)-\beta_{i, 1}-\beta_{i, 3}$

$\beta_{i, 1}$ differs from $\alpha_{i, 1}$ only in the term $\omega_{i}(-2) f_{i-2}(i-2, i-1)$. We explain only this term. Starting from $i$, with probability $\omega_{i}(-2)$, the walk jumps down from $i$ to $i-2$ and the excursion begins. Recall that $\beta_{i, 1}$ is the index corresponding to a $\mathscr{B}_{i, 1}$ excursion. Since a $\mathscr{B}_{i, 1}$ excursion ends with a jump $\{i-1 \rightarrow i\}$, after visiting $i-2$, it will reach $i-1$ from below before it comes to end. The sum of products of transition probabilities of all possible paths from $i-2$ to hit $i-1$ from below is $f_{i-2}(i-2, i-1)$.

One calculates from (4) that

$$
\begin{aligned}
\beta_{i, 1} & =\frac{\omega_{i}(-2) f_{i-2}(i-2, i-1) \omega_{i-1}(1)}{1-\omega_{i-1}(-1) f_{i-2}(i-2, i-1)-\omega_{i-1}(-2) f_{i-3}(i-2, i-1)}, \\
\beta_{i, 3} & =\frac{\omega_{i}(-2) f_{i-2}(i-2, i-1) \omega_{i-1}(2)}{1-\omega_{i-1}(-1) f_{i-2}(i-2, i-1)-\omega_{i-1}(-2) f_{i-3}(i-2, i-1)}
\end{aligned}
$$

At last, we consider type- $\mathscr{C}$ excursion (see (c) of Definition 1.1). Note that a type- $\mathscr{C}$ excursion at $i$ begins when the walk jumps down from $i+1$ to $i-1$. After that the walk runs in $(-\infty, i-1]$. At last the walk hits $[i, \infty)$ at some $j$ and the excursion goes to end.

Further, we define some indexes $\gamma_{i, 1}, \gamma_{i, 2}$, and $\gamma_{i, 3}$ correspondingly to $\mathscr{C}_{i, 1}, \mathscr{C}_{i, 3}$, and $\mathscr{C}_{i, 2}$. Let

$$
\begin{aligned}
& \gamma_{i, 1}:=\omega_{i+1}(-2) \sum_{n, m \geqslant 0} \frac{(n+m) !}{n ! m !}\left[\omega_{i-1}(-1) f_{i-2}(i-2, i-1)\right]^{n} \\
& {\left[\omega_{i-1}(-2) f_{i-3}(i-2, i-1)\right]^{m} \omega_{i-1}(1), } \\
& \gamma_{i, 3}:=\omega_{i+1}(-2) \sum_{n, m \geqslant 0} \frac{(n+m) !}{n ! m !}\left[\omega_{i-1}(-1) f_{i-2}(i-2, i-1)\right]^{n} \\
& {\left[\omega_{i-1}(-2) f_{i-3}(i-2, i-1)\right]^{m} \omega_{i-1}(2), } \\
& \gamma_{i, 2}:=\omega_{i+1}(-2)-\gamma_{i, 1}-\gamma_{i, 3} .
\end{aligned}
$$

A $\mathscr{C}_{i, 1}$ excursion differs from an $\mathscr{A}_{i, 1}$ excursion only in the first step. The first term in the product of $\gamma_{i, 1}$ is $\omega_{i+1}(-2)$. It means that with probability $\omega_{i+1}(-2)$, the walk jumps down from $i+1$ to $i-1$, and the excursion begins. 
$\gamma_{i, 2}$ and $\gamma_{i, 3}$ could be understood analogously to $\alpha_{i, 2}$ and $\alpha_{i, 3}$. We will not repeat them here. One follows from (5) that

$$
\begin{aligned}
\gamma_{i, 1} & =\frac{\omega_{i+1}(-2) \omega_{i-1}(1)}{1-\omega_{i-1}(-1) f_{i-2}(i-2, i-1)-\omega_{i-1}(-2) f_{i-3}(i-2, i-1)}, \\
\gamma_{i, 3} & =\frac{\omega_{i+1}(-2) \omega_{i-1}(2)}{1-\omega_{i-1}(-1) f_{i-2}(i-2, i-1)-\omega_{i-1}(-2) f_{i-3}(i-2, i-1)}
\end{aligned}
$$

Further, we show by path decomposition that $\left\{U_{i}\right\}_{i \leqslant 0}$ forms a nonhomogeneous multitype branching process.

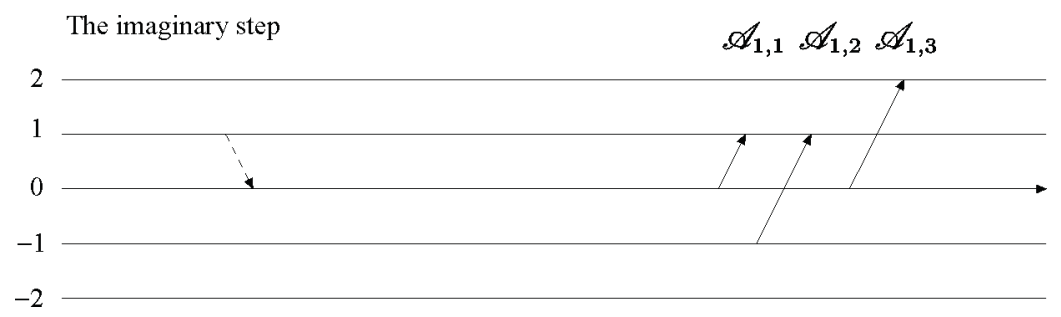

Fig. 2. The figure illustrates the immigration of the braching process. Adding the imaginary step $\{1 \rightarrow 0\}$, the path of the walk before $T_{1}$ forms an $\mathscr{A}$ excursion at 1 . It may be an $\mathscr{A}_{1,1}, \mathscr{A}_{1,2}$, or $\mathscr{A}_{1,3}$ excursion

Firstly, the branching process needs an ancestor (some particle immigrating in). The walk starts from 0 . But before $T_{1}$, there is no jump down from above 1 to 0 by the walk. One can imagine that there is a step by the walk from 1 to 0 before it starts from 0 that is, set $X_{-1}=1$. (One can also imagine that this step is from 2 to 0 . But this makes no difference.) Adding this imaginary step, the path $\left\{X_{-1}=1, X_{0}=0, X_{1}, \ldots, X_{T_{1}}\right\}$ forms a type- $\mathscr{A}$ excursion at 1 such that $A_{1,1}+A_{1,2}+A_{1,3}=1$.

The distributions of $A_{1,1}, A_{1,2}$, and $A_{1,3}$ are

$$
\begin{aligned}
& P_{\omega}^{0}\left(A_{1,1}=1\right)=\frac{\alpha_{1,1}}{\alpha_{1,1}+\alpha_{1,2}+\alpha_{1,3}} \\
& =\frac{\omega_{0}(1)}{1-\omega_{0}(-1) f_{-1}(-1,0)-\omega_{0}(-2) f_{-2}(-1,0)}, \\
& P_{\omega}^{0}\left(A_{1,3}=1\right)=\frac{\alpha_{1,3}}{\alpha_{1,1}+\alpha_{1,2}+\alpha_{1,3}} \\
& \quad=\frac{\omega_{0}(2)}{1-\omega_{0}(-1) f_{-1}(-1,0)-\omega_{0}(-2) f_{-2}(-1,0)}, \\
& P_{\omega}^{0}\left(A_{1,2}=1\right)=\frac{\alpha_{1,2}}{\alpha_{1,1}+\alpha_{1,2}+\alpha_{1,3}} \\
& \quad=\frac{\left[1-\omega_{0}(1)-\omega_{0}(2)-\omega_{0}(-1) f_{-1}(-1,0)-\omega_{0}(-2) f_{-2}(-1,0)\right]}{1-\omega_{0}(-1) f_{-1}(-1,0)-\omega_{0}(-2) f_{-2}(-1,0)} .
\end{aligned}
$$

The meaning of $P_{\omega}^{0}\left(A_{1,1}=1\right)$ is obvious. The total sum of the product of transition probabilities of all excursions of the form $\left\{X_{-1}=0, X_{0}=\right.$ 
$\left.1, X_{1}, \ldots, X_{T_{1}}\right\}$ is $\alpha_{1,1}+\alpha_{1,2}+\alpha_{1,3}=\omega_{1}(-1)$ and the total sum of the product of transition probabilities of all possible paths of an $\mathscr{A}_{1,1}$ excursion is $\alpha_{1,1}$. Therefore, $P_{\omega}^{0}\left(A_{1,1}=1\right)=\alpha_{1,1} /\left(\alpha_{1,1}+\alpha_{1,2}+\alpha_{1,3}\right)$. The values of $P_{\omega}^{0}\left(A_{1,2}=1\right)$ and $P_{\omega}^{0}\left(A_{1,3}=1\right)$ could be explained analogously.

We can treat the above discussed imaginary excursion as the particle immigrates in the branching system, say, the ancestor (immigration) of the branching process. The immigration laws are

$$
\begin{gathered}
P_{\omega}^{0}\left(U_{1}=(1,0, \ldots, 0)\right)=\frac{\omega_{0}(1)}{1-\omega_{0}(-1) f_{-1}(-1,0)-\omega_{0}(-2) f_{-2}(-1,0)}, \\
P_{\omega}^{0}\left(U_{1}=(0,1,0, \ldots, 0)\right)=\frac{\omega_{0}(2)}{1-\omega_{0}(-1) f_{-1}(-1,0)-\omega_{0}(-2) f_{-2}(-1,0)}, \\
P_{\omega}^{0}\left(U_{1}=(0,0,1,0, \ldots, 0)\right) \\
\quad=\frac{\left[1-\omega_{0}(1)-\omega_{0}(2)-\omega_{0}(-1) f_{-1}(-1,0)-\omega_{0}(-2) f_{-2}(-1,0)\right]}{1-\omega_{0}(-1) f_{-1}(-1,0)-\omega_{0}(-2) f_{-2}(-1,0)} .
\end{gathered}
$$

2.2. Branching mechanisms. After revealing the immigration law, we discuss the branching mechanism. Although there are 9 types of particles in the system, many of them share the same offspring distributions.

(a) Offspring distributions of $\mathscr{A}_{i+1,1}, \mathscr{A}_{i+1,3}, \mathscr{C}_{i+1,1}$, and $\mathscr{C}_{i+1,3}$ particles.

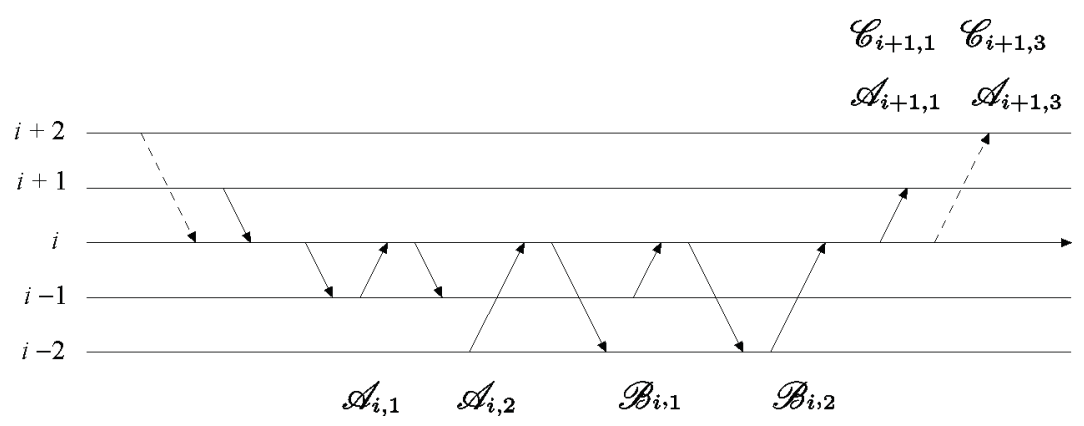

Fig. 3. The figure illustrates the offsprings of $\mathscr{A}_{i+1,1}, \mathscr{A}_{i+1,3}, \mathscr{C}_{i+1,1}$, and $\mathscr{C}_{i+1,3}$ particles. They could only give births to $\mathscr{A}_{i, 1}, \mathscr{A}_{i, 2}, \mathscr{B}_{i, 1}$, and $\mathscr{B}_{i, 2}$ particles.

For $i \leqslant 0$, conditioned on $U_{i+1}=\mathbf{e}_{1}$, that is, $\left\{A_{i+1,1}=1, A_{i+1,2}=\right.$ $\left.A_{i+1,3}=B_{i+1,1}=B_{i+1,2}=B_{i+1,3}=C_{i+1,1}=C_{i+1,2}=C_{i+1,3}=0\right\}$, we study the distribution of $U_{i}$. Note that the first step of excursion $\mathscr{A}_{i+1,1}$ is $\{i+1 \rightarrow i\}$ and the last step is $\{i \rightarrow i+1\}$. All contributions of this excursion to $U_{i}$ occurs between these two steps. Therefore this particle could give birthes only to excursions $\mathscr{A}_{i, 1}, \mathscr{A}_{i, 2}, \mathscr{B}_{i, 1}$, and $\mathscr{B}_{i, 2}$. We calculate the probability of the event $\left\{A_{i, 1}=a, A_{i, 2}=b, B_{i, 1}=c, B_{i, 2}=d\right\}$, that is, $\left\{U_{i}=\right.$ $(a, b, 0, c, d, 0,0,0,0)\}$, conditioned on $\left\{A_{i+1,1}=1\right\}\left(\right.$ Or $\left.U_{i+1}=\mathbf{e}_{1}\right)$. Indeed, one follows from the strong Markov property that those $a+b+c+d$ excursions at $i$ are independent, and the total number of all possible combinations 
of those excursions is $(a+b+c+d) ! / a ! b ! c ! d !$. To avoid heavy notation, we denote

$$
\zeta_{i}=1-\alpha_{i, 1}-\alpha_{i, 2}-\beta_{i, 1}-\beta_{i, 2}
$$

in the remainder of this section. Then we have that

$$
P_{\omega}^{0}\left(U_{i}=(a, b, 0, c, d, 0,0,0,0) \mid U_{i+1}=\mathbf{e}_{1}\right)=\frac{(a+b+c+d) !}{a ! b ! c ! d !} \alpha_{i, 1}^{a} \alpha_{i, 2}^{b} \beta_{i, 1}^{c} \beta_{i, 2}^{d} \zeta_{i} .
$$

One notes that $\mathscr{A}_{i+1,3}, \mathscr{C}_{i+1,1}, \mathscr{C}_{i+1,3}$, and $\mathscr{A}_{i+1,1}$ excursions share a common property, that is, the first step is from above $i$ to $i$ and the last step is from $i$ to above $i$. Therefore, they share the same offspring distribution. So analogously, one has

$$
\begin{array}{r}
P_{\omega}^{0}\left(U_{i}=(a, b, 0, c, d, 0,0,0,0) \mid U_{i+1}=\mathbf{e}_{3}\right) \\
=\frac{(a+b+c+d) !}{a ! b ! c ! d !} \alpha_{i, 1}^{a} \alpha_{i, 2}^{b} \beta_{i, 1}^{c} \beta_{i, 2}^{d} \zeta_{i}, \\
P_{\omega}^{0}\left(U_{i}=(a, b, 0, c, d, 0,0,0,0) \mid U_{i+1}=\mathbf{e}_{7}\right) \\
=\frac{(a+b+c+d) !}{a ! b ! c ! d !} \alpha_{i, 1}^{a} \alpha_{i, 2}^{b} \beta_{i, 1}^{c} \beta_{i, 2}^{d} \zeta_{i}, \\
P_{\omega}^{0}\left(U_{i}=(a, b, 0, c, d, 0,0,0,0) \mid U_{i+1}=\mathbf{e}_{9}\right) \\
=\frac{(a+b+c+d) !}{a ! b ! c ! d !} \alpha_{i, 1}^{a} \alpha_{i, 2}^{b} \beta_{i, 1}^{c} \beta_{i, 2}^{d} \zeta_{i} .
\end{array}
$$

(b) Offspring distributions of $\mathscr{A}_{i+1,2}$, and $\mathscr{C}_{i+1,2}$ particles. Conditioned on $\left\{U_{i+1}=\mathbf{e}_{2}\right\}$, that is, $\left\{A_{i+1,2}=1, A_{i+1,1}=A_{i+1,3}=B_{i+1,1}=B_{i+1,2}=\right.$ $\left.B_{i+1,3}=C_{i+1,1}=C_{i+1,2}=C_{i+1,3}=0\right\}$, we discuss the distribution of $U_{i}$. Recall that the first step of an $\mathscr{A}_{i+1,2}$ excursion is $\{i+1 \rightarrow i\}$ and the last step is $\{i-1 \rightarrow i+1\}$. Things get delicate because the last step $\{i-1 \rightarrow i+1\}$. Before the last step occurs, the walk must jump down from $i$, possibly to $i-1$ or $i-2$. If it jumps down from $i$ to $i-1$, it gives birth to an $\mathscr{A}_{i, 3}$ particle; if it jumps down from $i$ to $i-2$, it gives birth to a $\mathscr{B}_{i, 3}$ particle. That is $P_{\omega}^{0}\left(\mathscr{A}_{i, 3}+\mathscr{B}_{i, 3}=1 \mid U_{i+1}=\mathbf{e}_{2}\right)=1$.

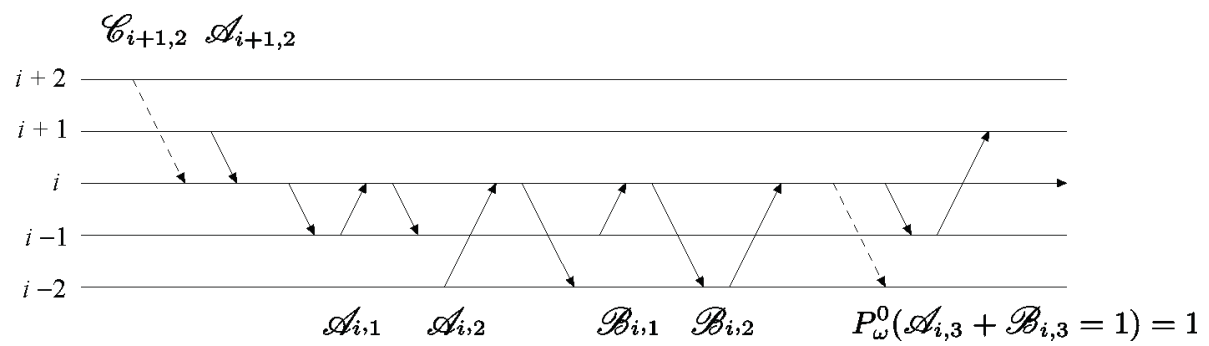

Fig. 4. The figure illustrates the offsprings of $\mathscr{A}_{i+1,2}, \mathscr{A}_{i+1,2}$. Before the last step $\{i-1 \rightarrow$ $i+1\}$ happens, with probability 1 , a $\mathscr{B}_{i, 3}$ or $\mathscr{A}_{i, 3}$ excursion would be born. 
The sum of the product of the transition probabilities of all possible paths of an excursion $\mathscr{A}_{i, 3}$ is $\alpha_{i, 3}$, and that of a $\mathscr{B}_{i, 3}$ excursion is $\beta_{i, 3}$. In this point of view, one concludes that

$$
P_{\omega}^{0}\left(A_{i, 3}=1 \mid U_{i+1}=\mathbf{e}_{2}\right)=1-P_{\omega}^{0}\left(B_{i, 3}=1 \mid U_{i+1}=\mathbf{e}_{2}\right)=\frac{\alpha_{i, 3}}{\alpha_{i, 3}+\beta_{i, 3}} .
$$

Before giving birth to the above discussed particle $\mathscr{B}_{i, 3}$ or $\mathscr{A}_{i, 3}$, the excursion $\mathscr{A}_{i+1,2}$ may give birthes to a number of $\mathscr{A}_{i, 1}, \mathscr{A}_{i, 2}, \mathscr{B}_{i, 1}$, and $\mathscr{B}_{i, 2}$ particles. Once again, one follows by path decomposition and Markov property that, all excursions born to particle $\mathscr{A}_{i+1,2}$ are independent. Therefore one has

$$
\begin{aligned}
& P_{\omega}^{0}\left(U_{i}=(a, b, 1, c, d, 0,0,0,0) \mid U_{i+1}=\mathbf{e}_{2}\right) \\
& \quad=\frac{(a+b+c+d) !}{a ! b ! c ! d !} \alpha_{i, 1}^{a} \alpha_{i, 2}^{b} \beta_{i, 1}^{c} \beta_{i, 2}^{d} \zeta_{i} \frac{\alpha_{i, 3}}{\alpha_{i, 3}+\beta_{i, 3}} \\
& P_{\omega}^{0}\left(U_{i}=(a, b, 0, c, d, 1,0,0,0) \mid U_{i+1}=\mathbf{e}_{2}\right) \\
& \quad=\frac{(a+b+c+d) !}{a ! b ! c ! d !} \alpha_{i, 1}^{a} \alpha_{i, 2}^{b} \beta_{i, 1}^{c} \beta_{i, 2}^{d} \zeta_{i} \frac{\beta_{i, 3}}{\alpha_{i, 3}+\beta_{i, 3}}
\end{aligned}
$$

Also, analogously, the offspring distributions of a $\mathscr{C}_{i+1,2}$ particle are

$$
\begin{aligned}
& P_{\omega}^{0}\left(U_{i}=(a, b, 1, c, d, 0,0,0,0) \mid U_{i+1}=\mathbf{e}_{8}\right) \\
& \quad=\frac{(a+b+c+d) !}{a ! b ! c ! d !} \alpha_{i, 1}^{a} \alpha_{i, 2}^{b} \beta_{i, 1}^{c} \beta_{i, 2}^{d} \zeta_{i} \frac{\alpha_{i, 3}}{\alpha_{i, 3}+\beta_{i, 3}} \\
& P_{\omega}^{0}\left(U_{i}=(a, b, 0, c, d, 1,0,0,0) \mid U_{i+1}=\mathbf{e}_{8}\right) \\
& \quad=\frac{(a+b+c+d) !}{a ! b ! c ! d !} \alpha_{i, 1}^{a} \alpha_{i, 2}^{b} \beta_{i, 1}^{c} \beta_{i, 2}^{d} \zeta_{i} \frac{\beta_{i, 3}}{\alpha_{i, 3}+\beta_{i, 3}} .
\end{aligned}
$$

(c) Offspring distributions of $\mathscr{B}_{i+1,1}$, and $\mathscr{B}_{i+1,3}$ particles $\mathscr{B}_{i+1,1}$ and $\mathscr{B}_{i+1,3}$ excursions differ from each other only in the last step. But their last steps are both from $i$ to above $i$. Therefore they have the same offspring distributions. Conditioned on $U_{i+1}=\mathbf{e}_{4}$, that is, $\left\{B_{i+1,1}=1, A_{i+1,1}=\right.$ $\left.A_{i+1,2}=A_{i+1,3}=B_{i+1,2}=B_{i+1,3}=C_{i+1,1}=C_{i+1,2}=C_{i+1,3}=0\right\}$, we discuss the offspring distributions of $\mathscr{B}_{i+1,1}$ particle. Note that an excursion $\mathscr{B}_{i+1,1}$ begins with a jump $\{i+1 \rightarrow i-1\}$ and ends with a jump $\{i \rightarrow i+1\}$. So after jumping down from $i+1$ to $i-1$, it must have an excursion returning to $i$. There are two possible approaches for the walk to return to $i$, that is, jumping from $i-1$ to $i$ or jumping from $i-2$ to $i$. From this point of view, one knows that a $\mathscr{B}_{i+1,1}$ particle gives birth to $\mathscr{C}_{i, 1}$ or $\mathscr{C}_{i, 2}$ particle at $i$ with probability 1 , and the approach the walk jumping from below $i$ to $i$ determines which particle will be born. Precisely, one has that

$$
P_{\omega}^{0}\left(C_{i, 1}+C_{i, 2}=1 \mid U_{i+1}=\mathbf{e}_{4}\right)=1 .
$$


Due to the same reason as (13),

$$
\begin{aligned}
& P_{\omega}^{0}\left(C_{i, 1}=1 \mid U_{i+1}=\mathbf{e}_{4}\right)=1-P_{\omega}^{0}\left(C_{i, 2}=1 \mid U_{i+1}=\mathbf{e}_{4}\right)=\frac{\gamma_{i, 1}}{\gamma_{i, 1}+\gamma_{i, 2}} . \\
& \begin{array}{l}
i+2 \\
i+1 \\
i-1 \\
i-2
\end{array} \\
& P_{\omega}^{0}\left(\mathscr{C}_{i, 1}+\mathscr{C}_{i, 2}=1\right)=1 \quad \mathscr{A}_{i, 1} \quad \mathscr{A}_{i, 2} \quad \mathscr{B}_{i, 1} \quad \mathscr{B}_{i, 2}
\end{aligned}
$$

Fig. 5. The figure illustrates the offsprings of $\mathscr{B}_{i+1,1}$ and $\mathscr{B}_{i+1,3}$. Since at last, the walk jumps from $i$ to some position above $i$, before the last step happens, it must return to $i$ from below. Therefore with probability $1, \mathscr{C}_{i, 1}$ or $\mathscr{C}_{i, 2}$ excursion would be born.

After giving birth to $\mathscr{C}_{i, 1}$ or $\mathscr{C}_{i, 2}$ particle, $\mathscr{B}_{i+1,1}$ particle may give births to certain number of $\mathscr{A}_{i, 1}, \mathscr{A}_{i, 2}, \mathscr{B}_{i, 1}$, and $\mathscr{B}_{i, 2}$ excursions. Markov property implies the independence of those born excursions. One has

$$
\begin{aligned}
& P_{\omega}^{0}\left(U_{i}=(a, b, 0, c, d, 0,1,0,0) \mid U_{i+1}=\mathbf{e}_{4}\right) \\
& \quad=\frac{(a+b+c+d) !}{a ! b ! c ! d !} \alpha_{i, 1}^{a} \alpha_{i, 2}^{b} \beta_{i, 1}^{c} \beta_{i, 2}^{d} \zeta_{i} \frac{\gamma_{i, 1}}{\gamma_{i, 1}+\gamma_{i, 2}}, \\
& P_{\omega}^{0}\left(U_{i}=(a, b, 0, c, d, 0,0,1,0) \mid U_{i+1}=\mathbf{e}_{4}\right) \\
& \quad=\frac{(a+b+c+d) !}{a ! b ! c ! d !} \alpha_{i, 1}^{a} \alpha_{i, 2}^{b} \beta_{i, 1}^{c} \beta_{i, 2}^{d} \zeta_{i} \frac{\gamma_{i, 2}}{\gamma_{i, 1}+\gamma_{i, 2}} .
\end{aligned}
$$

Analogously, the offspring distributions of a $\mathscr{B}_{i+1,3}$ particle are

$$
\begin{aligned}
& P_{\omega}^{0}\left(U_{i}=(a, b, 0, c, d, 0,1,0,0) \mid U_{i+1}=\mathbf{e}_{6}\right) \\
& \quad=\frac{(a+b+c+d) !}{a ! b ! c ! d !} \alpha_{i, 1}^{a} \alpha_{i, 2}^{b} \beta_{i, 1}^{c} \beta_{i, 2}^{d} \zeta_{i} \frac{\gamma_{i, 1}}{\gamma_{i, 1}+\gamma_{i, 2}}, \\
& P_{\omega}^{0}\left(U_{i}=(a, b, 0, c, d, 0,0,1,0) \mid U_{i+1}=\mathbf{e}_{6}\right) \\
& \quad=\frac{(a+b+c+d) !}{a ! b ! c ! d !} \alpha_{i, 1}^{a} \alpha_{i, 2}^{b} \beta_{i, 1}^{c} \beta_{i, 2}^{d} \zeta_{i} \frac{\gamma_{i, 2}}{\gamma_{i, 1}+\gamma_{i, 2}} .
\end{aligned}
$$

(d) Offspring distribution of $\mathscr{B}_{i+1,2}$ particles. Next conditioned on $U_{i+1}=\mathbf{e}_{5}$, that is, $\left\{B_{i+1,2}=1, A_{i+1,1}=A_{i+1,2}=A_{i+1,3}=B_{i+1,1}=B_{i+1,3}=\right.$ $\left.C_{i+1,1}=C_{i+1,2}=C_{i+1,3}=0\right\}$, we consider the offspring distributions of $\mathscr{B}_{i+1,2}$ particles. Special attention should be paid to $\mathscr{B}_{i+1,2}$ excursions. An excursion $\mathscr{B}_{i+1,2}$ begins with a jump $\{i+1 \rightarrow i-1\}$ and ends with a jump $\{i-1 \rightarrow i+1\}$. The point is whether it visited $i$ between the first and the last step. If it did not visit $i$ before the last step, then the excursion gives birth to a $\mathscr{C}_{i, 3}$ particle with probability 1 and generates no any other particle. If 
it did visit $i$ before the last step, that is, conditioned on $\left\{C_{i, 3}=0\right\}$, things get more complicated. Conditioned on $\left\{C_{i, 3}=0\right\}$, after jumping down from $i+1$ to $i-1$, the walk reaches $i$ from below $i$ at least one time, that is, from $i-1$ to $i$ or from $i-2$ to $i$. Therefore, one has

$$
P_{\omega}^{0}\left(C_{i, 1}+C_{i, 2}=1 \mid U_{i+1}=\mathbf{e}_{5}, C_{i, 3}=0\right)=1,
$$

and similarly as (13) that

$$
\begin{aligned}
& P_{\omega}^{0}\left(C_{i, 1}=1 \mid U_{i+1}=\mathbf{e}_{5}, C_{i, 3}=0\right) \\
& \quad=1-P_{\omega}^{0}\left(C_{i, 2}=1 \mid U_{i+1}=\mathbf{e}_{5}, C_{i, 3}=0\right)=\frac{\gamma_{i, 1}}{\gamma_{i, 1}+\gamma_{i, 2}}
\end{aligned}
$$

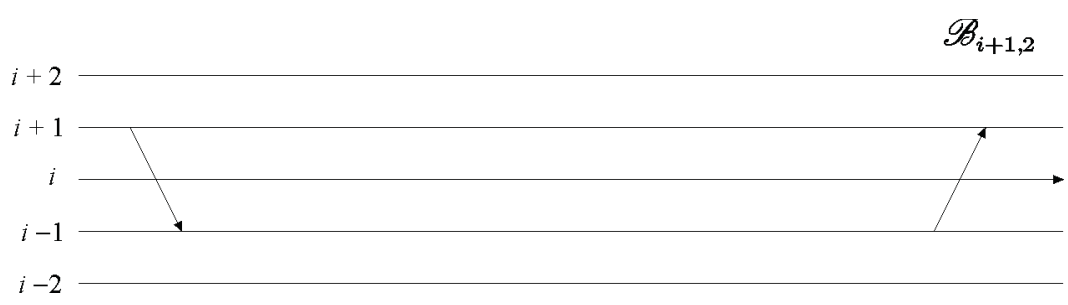

Fig. 6a. The figure illustrates the offsprings (case 1 ) of $\mathscr{B}_{i+1,2}$ excursion. The walk never visited $i$ between the first and the last step. Theofore, only a type $\mathscr{C}_{i, 3}$ excursion would be born.

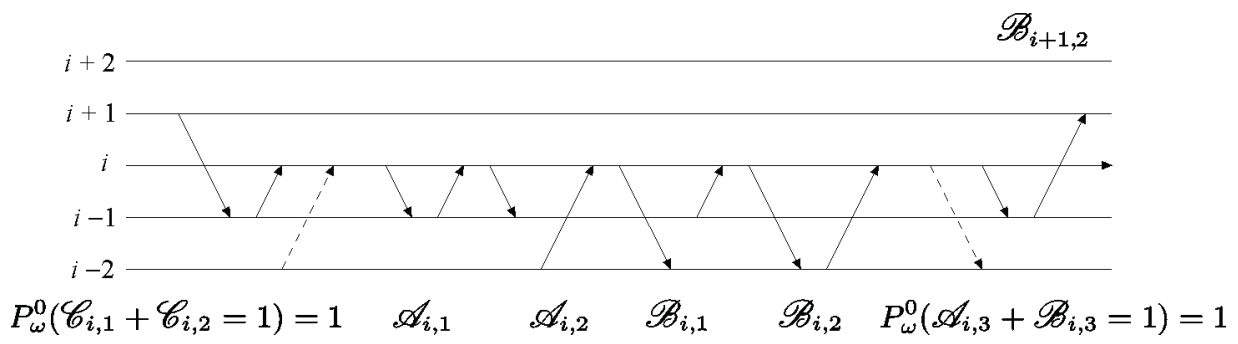

Fig. 6b. The figure illustrates the offsprings (case 2) of $\mathscr{B}_{i+1,2}$ excursion. Between the first and the last step, the walk did visit $i$. Therefore, $P_{\omega}^{0}\left(\mathscr{C}_{i, 1}+\mathscr{C}_{i, 2}=1\right)=1$ and $P_{\omega}^{0}\left(\mathscr{A}_{i, 3}+\mathscr{B}_{i, 3}=1\right)=1$.

Since the last step of excursion $\mathscr{B}_{i+1,2}$ is from $i-1$ to $i+1$, conditioned on $\left\{U_{i+1}=\mathbf{e}_{5}, C_{i, 3}=0\right\}$, after reaching $i$ from below, it must jump down from $i$ to $i-1$ or from $i$ to $i-2$ at least one time, before the last step $\{i-1 \rightarrow i+1\}$ occurs. In the other words,

$$
\begin{aligned}
& P_{\omega}^{0}\left(A_{i, 3}+B_{i, 3}=1 \mid U_{i+1}=\mathbf{e}_{5}, C_{i, 3}=0\right)=1 \\
& P_{\omega}^{0}\left(A_{i, 3}=1 \mid U_{i+1}=\mathbf{e}_{5}, C_{i, 3}=0\right) \\
& \quad=1-P_{\omega}^{0}\left(B_{i, 2}=1 \mid U_{i+1}=\mathbf{e}_{5}, C_{i, 3}=0\right)=\frac{\alpha_{i, 3}}{\alpha_{i, 3}+\beta_{i, 3}} .
\end{aligned}
$$


On the other hand, conditioned on $\left\{U_{i+1}=\mathbf{e}_{5}, C_{i, 3}=0\right\}$, besides giving birthes to the above discussed particles, it may give birthes to a number of $\mathscr{A}_{i, 1}, \mathscr{A}_{i, 2}, \mathscr{B}_{i, 1}$, and $\mathscr{B}_{i, 2}$ particles.

Further, we calculate the probabilities of $\left\{C_{i, 3}=1\right\}$ and $\left\{C_{i, 3}=0\right\}$ conditioned on $\left\{U_{i+1}=\mathbf{e}_{5}\right\}$.

One follows from the above discussions that the sum of the products of transition probabilities of all possible paths of an excursion $\mathscr{B}_{i+1,2}$ is $\beta_{i+1,2}$ and all those possible paths could be divided into two classes. Paths of the first class never visited $i$ and paths of the second class visited $i$ from below certain times. Sum of the product of transition probabilities of all possible first kind paths is $\gamma_{i, 3}$ and that of the second kind paths is $\beta_{i+1,2}-\gamma_{i, 3}$. Therefore,

$$
P_{\omega}^{0}\left(C_{i, 3}=1 \mid U_{i+1}=\mathbf{e}_{5}\right)=1-P_{\omega}^{0}\left(C_{i, 3}=0 \mid U_{i+1}=\mathbf{e}_{5}\right)=\frac{\gamma_{i, 3}}{\beta_{i+1,2}} .
$$

Also the Markov property implies the independence of all excursions born to $\mathscr{B}_{i+1,2}$ particle at $i$. One follows by path decomposition and independence that

$$
\begin{aligned}
& P_{\omega}^{0}\left(U_{i}=(0, \ldots, 0,1) \mid U_{i+1}=\mathbf{e}_{5}\right)=\frac{\gamma_{i, 3}}{\beta_{i+1,2}}, \\
& P_{\omega}^{0}\left(U_{i}=(a, b, 1, c, d, 0,1,0,0) \mid U_{i+1}=\mathbf{e}_{5}\right) \\
& =\frac{(a+b+c+d) !}{a ! b ! c ! d !} \alpha_{i, 1}^{a} \alpha_{i, 2}^{b} \beta_{i, 1}^{c} \beta_{i, 2}^{d} \zeta_{i} \frac{\left(\beta_{i+1,2}-\gamma_{i, 3}\right) \gamma_{i, 1} \alpha_{i, 3}}{\beta_{i+1,2}\left(\gamma_{i, 1}+\gamma_{i, 2}\right)\left(\alpha_{i, 3}+\beta_{i, 3}\right)}, \\
& P_{\omega}^{0}\left(U_{i}=(a, b, 1, c, d, 0,0,1,0) \mid U_{i+1}=\mathbf{e}_{5}\right) \\
& =\frac{(a+b+c+d) !}{a ! b ! c ! d !} \alpha_{i, 1}^{a} \alpha_{i, 2}^{b} \beta_{i, 1}^{c} \beta_{i, 2}^{d} \zeta_{i} \frac{\left(\beta_{i+1,2}-\gamma_{i, 3}\right) \gamma_{i, 2} \alpha_{i, 3}}{\beta_{i+1,2}\left(\gamma_{i, 1}+\gamma_{i, 2}\right)\left(\alpha_{i, 3}+\beta_{i, 3}\right)}, \\
& P_{\omega}^{0}\left(U_{i}=(a, b, 0, c, d, 1,1,0,0) \mid U_{i+1}=\mathbf{e}_{5}\right) \\
& =\frac{(a+b+c+d) !}{a ! b ! c ! d !} \alpha_{i, 1}^{a} \alpha_{i, 2}^{b} \beta_{i, 1}^{c} \beta_{i, 2}^{d} \zeta_{i} \frac{\left(\beta_{i+1,2}-\gamma_{i, 3}\right) \gamma_{i, 1} \beta_{i, 3}}{\beta_{i+1,2}\left(\gamma_{i, 1}+\gamma_{i, 2}\right)\left(\alpha_{i, 3}+\beta_{i, 3}\right)}, \\
& P_{\omega}^{0}\left(U_{i}=(a, b, 0, c, d, 1,0,1,0) \mid U_{i+1}=\mathbf{e}_{5}\right) \\
& =\frac{(a+b+c+d) !}{a ! b ! c ! d !} \alpha_{i, 1}^{a} \alpha_{i, 2}^{b} \beta_{i, 1}^{c} \beta_{i, 2}^{d} \zeta_{i} \frac{\left(\beta_{i+1,2}-\gamma_{i, 3}\right) \gamma_{i, 2} \beta_{i, 3}}{\beta_{i+1,2}\left(\gamma_{i, 1}+\gamma_{i, 2}\right)\left(\alpha_{i, 3}+\beta_{i, 3}\right)} .
\end{aligned}
$$

Summing up the discussions of this section, we have the following theorem, which has also been stated as Theorem 1.2 in the introduction section.

Theorem 2.1. Suppose that $\lim \sup _{n \rightarrow \infty} X_{n}=\infty$. Then $\left\{U_{i}\right\}_{i \leqslant 1}$ is a 9 type nonhomogeneous branching process with immigration distribution as in (6), (7), and (8) above, and offsprings distributions as in (9)-(12), (14)-(17), (18)-(21), and (22)-(26) above.

Corollary 2.1. Let $Q_{i}$ be a $9 \times 9$ matrix, whose lth row is means of the number of particles born to a type-l particle of the $(i+1)$ th gen- 
eration. The matrices $Q_{i}$ are called the mean matrices of the branching process $\left\{U_{i}\right\}_{i \leqslant 1}$. Let $x_{i}=\alpha_{i, 1} / 1-\alpha_{i, 1}-\alpha_{i, 2}-\beta_{i, 1}-\beta_{i, 2}, \quad y_{i}=$ $\alpha_{i, 2} / 1-\alpha_{i, 1}-\alpha_{i, 2}-\beta_{i, 1}-\beta_{i, 2}, z_{i}=\beta_{i, 1} / 1-\alpha_{i, 1}-\alpha_{i, 2}-\beta_{i, 1}-\beta_{i, 2}, w_{i}=$ $\beta_{i, 2} / 1-\alpha_{i, 1}-\alpha_{i, 2}-\beta_{i, 1}-\beta_{i, 2}, 1-v=\gamma_{i, 3} / \beta_{i+1,2}, s_{i}=\alpha_{i, 3} / \alpha_{i, 3}+\beta_{i, 3}$ and $t_{i}=\gamma_{i, 1} / \gamma_{i, 1}+\gamma_{i, 2}$. Then one calculates from the branching mechanism of $\left\{U_{i}\right\}_{i \leqslant 1}$ that

$$
Q_{i}=\left(\begin{array}{ccccccccc}
x_{i} & y_{i} & 0 & z_{i} & w_{i} & 0 & 0 & 0 & 0 \\
x_{i} & y_{i} & s_{i} & z_{i} & w_{i} & 1-s_{i} & 0 & 0 & 0 \\
x_{i} & y_{i} & 0 & z_{i} & w_{i} & 0 & 0 & 0 & 0 \\
x_{i} & y_{i} & 0 & z_{i} & w_{i} & 0 & t_{i} & 1-t_{i} & 0 \\
x_{i} v_{i} & y_{i} v_{i} & s_{i} v_{i} & z_{i} v_{i} & w_{i} v_{i} & \left(1-s_{i}\right) v_{i} & t_{i} v_{i} & \left(1-t_{i}\right) v_{i} & 1-v_{i} \\
x_{i} & y_{i} & 0 & z_{i} & w_{i} & 0 & t_{i} & 1-t_{i} & 0 \\
x_{i} & y_{i} & 0 & z_{i} & w_{i} & 0 & 0 & 0 & 0 \\
x_{i} & y_{i} & s_{i} & z_{i} & w_{i} & 1-s_{i} & 0 & 0 & 0 \\
x_{i} & y_{i} & 0 & z_{i} & w_{i} & 0 & 0 & 0 & 0
\end{array}\right)
$$

We remark that the sequence $\left\{Q_{i}\right\}$ will be used to derive the velocity of (2-2) RWRE in what follows.

3. The ladder time $T_{1}$ and the branching process. Proof of Theorem 1.1. Recall that $T_{1}=\inf \left\{n \geqslant 0: X_{n}>0\right\}$ is the first hitting time of $[1, \infty)$. In this section, we aim at expressing $T_{1}$ in terms of the multitype branching process $\left\{U_{i}\right\}_{i \leqslant 1}$. Define

$D_{i, 1}=\#\left\{\right.$ steps by the walk from above $i-1$ to $i-1$ before time $\left.T_{1}\right\}$,

$V_{i, 1}=\#\left\{\right.$ steps by the walk from $i-1$ to $i$ before time $\left.T_{1}\right\}$,

$V_{i, 2}=\#\left\{\right.$ steps by the walk from $i-2$ to $i$ before time $\left.T_{1}\right\}$.

Then $\sum_{i \leqslant 0} D_{i, 1}$ counts all steps jumping downward by the walk before time $T_{1}$ and $\sum_{i \leqslant 0} V_{i, 1}+V_{i, 2}$ counts all steps jumping upward by the walk before time $T_{1}$. But both $\sum_{i \leqslant 0} D_{i, 1}$ and $\sum_{i \leqslant 0} V_{i, 1}+V_{i, 2}$ did not count the last step by the walk hitting $[1, \infty)$. Therefore,

$$
T_{1}=1+\sum_{i \leqslant 0} D_{i, 1}+V_{i, 1}+V_{i, 2}
$$

This together with the fact $D_{i, 1}=A_{i, 1}+A_{i, 2}+A_{i, 3}+C_{i, 1}+C_{i, 2}+C_{i, 3}$, $V_{i, 1}=A_{i, 1}+B_{i, 1}+C_{i, 1}$, and $V_{i, 2}=A_{i, 2}+B_{i, 2}+C_{i, 2}$ implies that

$$
\begin{aligned}
T_{1} & =1+\sum_{i \leqslant 0} 2 A_{i, 1}+2 A_{i, 2}+A_{i, 3}+B_{i, 1}+B_{i, 2}+2 C_{i, 1}+2 C_{i, 2}+C_{i, 3} \\
& =1+\sum_{i \leqslant 0} U_{i}(2,2,1,1,1,0,2,2,1)^{\mathrm{T}} .
\end{aligned}
$$


Further, we calculate the moment of $T_{1}$ by mean of the branching process. One calculates from the immigration distributions (6)-(8) that

$$
\begin{aligned}
& E_{\omega}^{0}\left(U_{1}\right) \\
& =\left(\frac{\alpha_{1,1}}{\alpha_{1,1}+\alpha_{1,2}+\alpha_{1,3}}, \frac{\alpha_{1,2}}{\alpha_{1,1}+\alpha_{1,2}+\alpha_{1,3}}, \frac{\alpha_{1,3}}{\alpha_{1,1}+\alpha_{1,2}+\alpha_{1,3}}, 0, \ldots, 0\right) \\
& =: u_{1} .
\end{aligned}
$$

Therefore, one follows from Markov property that, for $i \leqslant 0, E_{\omega}^{0}\left(U_{i}\right)=$ $u_{1} Q_{0} \cdots Q_{i}$. Substituting to (28) one has that

$$
E_{\omega}^{0}\left(T_{1}\right)=1+\sum_{i \leqslant 0} u_{1} Q_{0} \cdots Q_{i}(2,2,1,1,1,0,2,2,1)^{\mathrm{T}} .
$$

Theorem 1.1 is proved.

4. Invariant measure equation and LLN of (2-2) RWRE. Proof of Theorem 1.3. In this section, we consider random walk $\left\{X_{n}\right\}$ in random environment $\omega$. Since by assumption $E^{0}\left(T_{1}\right)<\infty$, then $P^{0}$-a.s., $T_{1}<\infty$.

Define $\bar{\omega}(n)=\theta^{X_{n}} \omega$. The process $\{\bar{\omega}(n)\}$ is called the environment viewed from particles. One easily shows that $\{\bar{\omega}(n)\}$ is indeed a Markov process under either $P_{\omega}^{0}$ or $P^{0}$, with transitional kernel

$K\left(\omega, \mathrm{d} \omega^{\prime}\right)=\omega_{0}(2) \delta_{\theta^{2} \omega=\omega^{\prime}}+\omega_{0}(1) \delta_{\theta \omega=\omega^{\prime}}+\omega_{0}(-1) \delta_{\theta^{-1} \omega=\omega^{\prime}}+\omega_{0}(-2) \delta_{\theta^{-2} \omega=\omega^{\prime}}$.

It is important to find the invariant measure and the corresponding invariant density for the transition kernel $K\left(\omega, \mathrm{d} \omega^{\prime}\right)$. If one has the invariant density in the hand, then one could show the LLN for (2-2) RWRE $\left\{X_{n}\right\}$ and the limit velocity of the transient walk could be expressed by the invariant density.

We borrow some notation from [4] to give the invariant measure. Define $\varphi_{\theta^{k} \omega}^{1}=P_{\theta^{k} \omega}^{0}\left(X_{T_{1}}=1\right)$ and $\varphi_{\theta^{k} \omega}^{2}=P_{\theta^{k} \omega}^{0}\left(X_{T_{1}}=2\right)$. Whenever $E^{0}\left(T_{1}\right)<\infty$, define

$$
Q(\mathrm{~d} \omega)=E^{0}\left(\frac{\mathbf{1}_{X_{T_{1}}=1}}{\varphi_{\omega}^{1}} \sum_{i=0}^{T_{1}-1} \mathbf{1}_{\bar{\omega}(i) \in \mathrm{d} \omega}+\frac{\mathbf{1}_{X_{T_{1}}=2}}{\varphi_{\omega}^{2}} \sum_{i=0}^{T_{1}-1} \mathbf{1}_{\bar{\omega}(i) \in \mathrm{d} \omega}\right)
$$

and

$$
\bar{Q}(\mathrm{~d} \omega)=\frac{\mathrm{Q}(\mathrm{d} \omega)}{\mathrm{Q}(\Omega)} .
$$

Further, we show that the measure $Q$ is invariant under transition kernel $K\left(\omega, \mathrm{d} \omega^{\prime}\right)$, that is,

$$
Q(B)=\iint \mathbf{1}_{\omega^{\prime} \in B} K\left(\omega, \mathrm{d} \omega^{\prime}\right) \mathrm{Q}(\mathrm{d} \omega), \quad \text { for } \mathrm{B} \in \mathscr{F},
$$

and that

$$
\frac{\mathrm{dQ}}{\mathrm{d} \mathbf{P}}=\sum_{i \leqslant 0} E_{\theta^{-i} \omega}^{0}\left(N_{i} \mid X_{T_{1}}=1\right)+E_{\theta^{-i} \omega}^{0}\left(N_{i} \mid X_{T_{1}}=2\right),
$$

where $N_{i}:=\#\left\{k \in\left[0, T_{1}\right): X_{k}=i\right\}$. 
We remark that the proofs of (30) and (31) could be found in [4], but to make our paper more self-contained, we repeat the proofs here. To show (30), note that on one hand,

$$
\begin{aligned}
Q(B)= & \sum_{i=0}^{\infty} E^{0}\left(\frac{\mathbf{1}_{X_{T_{1}}=1}}{\varphi_{\omega}^{1}} ; T_{1}>i ; \bar{\omega}(i) \in B\right) \\
& +\sum_{i=0}^{\infty} E^{0}\left(\frac{\mathbf{1}_{X_{T_{1}}=2}}{\varphi_{\omega}^{2}} ; T_{1}>i ; \bar{\omega}(i) \in B\right),
\end{aligned}
$$

and on the other hand,

$$
\begin{aligned}
\iint \mathbf{1}_{\omega^{\prime} \in B} K\left(\omega, \mathrm{d} \omega^{\prime}\right) Q(\mathrm{~d} \omega)= & \sum_{i=0}^{\infty} E^{0}\left(\frac{\mathbf{1}_{X_{T_{1}}=1}}{\varphi_{\omega}^{1}} ; T_{1}>i ; \bar{\omega}(i+1) \in B\right) \\
& +\sum_{i=0}^{\infty} E^{0}\left(\frac{\mathbf{1}_{X_{T_{1}}=2}}{\varphi_{\omega}^{2}} ; T_{1}>i ; \bar{\omega}(i+1) \in B\right) \\
= & \sum_{j=1}^{\infty} E^{0}\left(\frac{\mathbf{1}_{X_{T_{1}}=1}}{\varphi_{\omega}^{1}} ; T_{1}>j ; \bar{\omega}(j) \in B\right) \\
& +\sum_{j=1}^{\infty} E^{0}\left(\frac{\mathbf{1}_{X_{T_{1}}}=2}{\varphi_{\omega}^{2}} ; T_{1}>j ; \bar{\omega}(j) \in B\right) \\
& +E^{0}\left(\frac{\mathbf{1}_{X_{T_{1}}=1}}{\varphi_{\omega}^{1}} ; T_{1}<\infty ; \bar{\omega}\left(T_{1}\right) \in B\right) \\
& +E^{0}\left(\frac{\mathbf{1}_{X_{T_{1}}=2}}{\varphi_{\omega}^{2}} ; T_{1}<\infty ; \bar{\omega}\left(T_{1}\right) \in B\right) .
\end{aligned}
$$

It remains to show

$$
E^{0}\left(\frac{\mathbf{1}_{X_{T_{1}}=i}}{\varphi_{\omega}^{i}} ; T_{1}>0 ; \bar{\omega}(0) \in B\right)=E^{0}\left(\frac{\mathbf{1}_{X_{T_{1}}=i}}{\varphi_{\omega}^{i}} ; T_{1}<\infty ; \bar{\omega}\left(T_{1}\right) \in B\right),
$$

where $i=1,2$. Indeed, for $i=1,2$, the right-hand side of (32) equals to

$$
\begin{aligned}
& E_{\mathbf{P}}\left(\frac{1}{\varphi_{\omega}^{i}} E_{\omega}^{0}\left(\mathbf{1}_{X_{T_{1}}=i} ; \bar{\omega}\left(T_{1}\right) \in B ; T_{1}<\infty\right)\right) \\
& \quad=E_{\mathbf{P}}\left(P_{\omega}^{0}\left(\bar{\omega}\left(T_{1}\right) \in B ; T_{1}<\infty \mid X_{T_{1}}=i\right)\right) \\
& \quad=E_{\mathbf{P}}\left(\mathbf{1}_{\theta^{i} \omega \in B} \cdot P_{\omega}^{0}\left(T_{1}<\infty \mid X_{T_{1}}=i\right)\right)=\mathbf{P}\left(\theta^{i} \omega \in B\right),
\end{aligned}
$$

where the last equation holds because $P_{\omega}^{0}\left(T_{1}<\infty\right)=1$. Similarly, the lefthand side of (32) equals to $\mathbf{P}(\omega \in B)$. Then (32) follows from the stationarity of the environment.

To prove (31), let $f: \Omega \rightarrow \mathbf{R}$ be any test function. Then,

$$
\begin{aligned}
\int f \mathrm{~d} Q & =E^{0}\left(\sum_{i=0}^{T_{1}-1} f(\bar{\omega}(i)) \frac{\mathbf{1}_{X_{T_{1}}=1}}{\varphi_{\omega}^{1}}+\sum_{i=0}^{T_{1}-1} f(\bar{\omega}(i)) \frac{\mathbf{1}_{X_{T_{1}}=2}}{\varphi_{\omega}^{2}}\right) \\
& =E^{0}\left(\sum_{i \leqslant 0} f\left(\theta^{i} \omega\right) N_{i} \frac{\mathbf{1}_{X_{T_{1}}=1}}{\varphi_{\omega}^{1}}+\sum_{i \leqslant 0} f\left(\theta^{i} \omega\right) N_{i} \frac{\mathbf{1}_{X_{T_{1}}=2}}{\varphi_{\omega}^{2}}\right) .
\end{aligned}
$$


Using the shift invariance of $\mathbf{P}$, we get

$$
\begin{aligned}
& \int f \mathrm{~d} Q=\sum_{i \leqslant 0} E_{\mathbf{P}}\left(f\left(\theta^{i} \omega\right)\left(\frac{1}{\varphi_{\omega}^{1}} E_{\omega}^{0}\left(N_{i} \cdot \mathbf{1}_{X_{T_{1}}=1}\right)+\frac{1}{\varphi_{\omega}^{2}} E_{\omega}^{0}\left(N_{i} \cdot \mathbf{1}_{X_{T_{1}}=2}\right)\right)\right) \\
& =\sum_{i \leqslant 0} E_{\mathbf{P}}\left(f(\omega)\left(\frac{1}{\varphi_{\theta^{-i} \omega}^{1}} E_{\theta^{-i} \omega}^{0}\left(N_{i} \cdot \mathbf{1}_{X_{T_{1}}=1}\right)+\frac{1}{\varphi_{\theta^{-i} \omega}^{2}} E_{\theta^{-i} \omega}^{0}\left(N_{i} \cdot \mathbf{1}_{X_{T_{1}}=2}\right)\right)\right) \\
& =E_{\mathbf{P}}\left(f(\omega) \sum_{i \leqslant 0}\left(\frac{1}{\varphi_{\theta^{-i} \omega}^{1}} E_{\theta^{-i} \omega}^{0}\left(N_{i} \cdot \mathbf{1}_{X_{T_{1}}=1}\right)+\frac{1}{\varphi_{\theta^{-i} \omega}^{2}} E_{\theta^{-i} \omega}^{0}\left(N_{i} \cdot \mathbf{1}_{X_{T_{1}}=2}\right)\right)\right) \\
& =E_{\mathbf{P}}\left(f(\omega) \sum_{i \leqslant 0}\left(E_{\theta^{-i} \omega}^{0}\left(N_{i} \mid X_{T_{1}}=1\right)+E_{\theta^{-i} \omega}^{0}\left(N_{i} \mid X_{T_{1}}=2\right)\right)\right),
\end{aligned}
$$

which proves $(31)$.

The branching structure enables us to calculate the right-hand side of (31) and give specifically $\mathrm{dQ} / \mathrm{d} \mathbf{P}$. In fact, note that for $i \leqslant-2$,

$$
\begin{aligned}
N_{i}= & A_{i+1,1}+A_{i+1,2}+A_{i+1,3}+C_{i+1,1}+C_{i+1,2}+C_{i+1,3} \\
& +A_{i, 1}+A_{i, 2}+B_{i, 1}+B_{i, 2}+C_{i, 1}+C_{i, 2} .
\end{aligned}
$$

Then

$$
\begin{aligned}
E_{\omega}^{0}\left(N_{i} \mid X_{T_{1}}=1\right)= & \frac{E_{\omega}^{0}\left(U_{i+1}(1,1,1,0,0,0,1,1,1)^{T} ; X_{T_{1}}=1\right)}{P_{\omega}^{0}\left(X_{T_{1}}=1\right)} \\
& +\frac{E_{\omega}^{0}\left(U_{i}(1,1,0,1,1,0,1,1,0)^{T} ; X_{T_{1}}=1\right)}{P_{\omega}^{0}\left(X_{T_{1}}=1\right)} .
\end{aligned}
$$

Temporarily, we set $\mathbf{v}_{1}=(1,1,1,0,0,0,1,1,1)^{\mathrm{T}}, \mathbf{v}_{2}=(1,1,0,1,1,0,1,1,0)^{\mathrm{T}}$. The first term in the right-hand side of (33) equals

$$
\begin{gathered}
\frac{1}{f_{0}(1)} E_{\omega}^{0}\left(U_{i+1} \mathbf{v}_{1} ; A_{1,1}=1\right)+\frac{1}{f_{0}(1)} E_{\omega}^{0}\left(U_{i+1} \mathbf{v}_{1} ; A_{1,2}=1\right) \\
=\frac{\omega_{1}(-1)}{\alpha_{1,1}+\alpha_{1,2}}\left(E_{\omega}^{0}\left(U_{i+1} \mathbf{v}_{1} \mid A_{1,1}=1\right) P_{\omega}^{0}\left(A_{1,1}=1\right)\right. \\
\left.\quad+E_{\omega}^{0}\left(U_{i+1} \mathbf{v}_{1} \mid A_{1,2}=1\right) P_{\omega}^{0}\left(A_{1,2}=1\right)\right) \\
=\frac{\omega_{1}(-1)}{\alpha_{1,1}+\alpha_{1,2}}\left(\frac{\alpha_{1,1}}{\alpha_{1,1}+\alpha_{1,2}+\alpha_{1,3}} \mathbf{e}_{1} Q_{0} \cdots Q_{i+1} \mathbf{v}_{1}\right. \\
\left.\quad+\frac{\alpha_{1,2}}{\alpha_{1,1}+\alpha_{1,2}+\alpha_{1,3}} \mathbf{e}_{2} Q_{0} \cdots Q_{i+1} \mathbf{v}_{1}\right) \\
=\left(\frac{\alpha_{1,1}}{\alpha_{1,1}+\alpha_{1,2}}, \frac{\alpha_{1,2}}{\alpha_{1,1}+\alpha_{1,2}}, 0, \ldots, 0\right) Q_{0} \cdots Q_{i+1} \mathbf{v}_{1},
\end{gathered}
$$

since $f_{0}(1)=\left(\alpha_{1,1}+\alpha_{1,2}\right) / \omega_{1}(-1)$ and $\alpha_{1,1}+\alpha_{1,2}+\alpha_{1,3}=\omega_{1}(-1)$.

Similarly the second term in the right-hand side of (33) equals

$$
\left(\frac{\alpha_{1,1}}{\alpha_{1,1}+\alpha_{1,2}}, \frac{\alpha_{1,2}}{\alpha_{1,1}+\alpha_{1,2}}, 0, \ldots, 0\right) Q_{0} \cdots Q_{i} \mathbf{v}_{2}
$$


Therefore,

$$
\begin{aligned}
E_{\omega}^{0}\left(N_{i} \mid X_{T_{1}}=1\right)= & \left(\frac{\alpha_{1,1}}{\alpha_{1,1}+\alpha_{1,2}}, \frac{\alpha_{1,2}}{\alpha_{1,1}+\alpha_{1,2}}, 0, \ldots, 0\right) \\
& \times\left(Q_{0} \cdots Q_{i+1} \mathbf{v}_{1}+Q_{0} \cdots Q_{i} \mathbf{v}_{2}\right)
\end{aligned}
$$

On the other hand,

$$
E_{\omega}^{0}\left(N_{i} \mid X_{T_{1}}=2\right)=E_{\omega}^{0}\left(N_{i} \mid A_{1,3}=1\right)=\mathbf{e}_{3}\left(Q_{0} \cdots Q_{i+1} \mathbf{v}_{1}+Q_{0} \cdots Q_{i} \mathbf{v}_{2}\right)
$$

Further, since $N_{0}=1+A_{0,1}+A_{0,2}+B_{0,1}+B_{0,2}$, it follows that

$$
\begin{aligned}
E_{\omega}^{0}\left(N_{0} \mid X_{T_{1}}=2\right)= & E_{\omega}^{0}\left(N_{0} \mid A_{1,3}=1\right) \\
= & 1+\mathbf{e}_{3} Q_{0}(1,1,0,1,1,0,0,0,0)^{\mathrm{T}}=1+\mathbf{e}_{3} Q_{0} \mathbf{v}_{2} \\
E_{\omega}^{0}\left(N_{0} \mid X_{T_{1}}=1\right)= & 1+\left(\frac{\alpha_{1,1}}{\alpha_{1,1}+\alpha_{1,2}}, \frac{\alpha_{1,2}}{\alpha_{1,1}+\alpha_{1,2}}, 0, \ldots, 0\right) \\
& \times Q_{0}(1,1,0,1,1,0,0,0,0)^{\mathrm{T}} \\
= & 1+\left(\frac{\alpha_{1,1}}{\alpha_{1,1}+\alpha_{1,2}}, \frac{\alpha_{1,2}}{\alpha_{1,1}+\alpha_{1,2}}, 0, \ldots, 0\right) Q_{0} \mathbf{v}_{2}
\end{aligned}
$$

where the second equality holds due to the special structure of the mean matrix $Q_{0}$. Note that

$$
N_{-1}=A_{0,1}+A_{0,2}+A_{0,3}+A_{-1,1}+A_{-1,2}+B_{-1,1}+B_{-1,2}+C_{-1,1}+C_{-1,2}
$$

where we mention that if the last step before $T_{1}$ is $\{-1 \rightarrow 1\}$, then $A_{0,3}=1$, otherwise, $A_{0,3}=0$.

Similarly as above, one has that

$$
\begin{aligned}
E_{\omega}^{0}\left(N_{-1} \mid X_{T_{1}}=1\right)= & \left(\frac{\alpha_{1,1}}{\alpha_{1,1}+\alpha_{1,2}}, \frac{\alpha_{1,2}}{\alpha_{1,1}+\alpha_{1,2}}, 0, \ldots, 0\right) \\
& \times\left(Q_{0}(1,1,1,0, \ldots, 0)^{\mathrm{T}}+Q_{0} Q_{-1} \mathbf{v}_{2}\right) \\
= & \left(\frac{\alpha_{1,1}}{\alpha_{1,1}+\alpha_{1,2}}, \frac{\alpha_{1,2}}{\alpha_{1,1}+\alpha_{1,2}}, 0, \ldots, 0\right) \\
& \times\left(Q_{0}(1,1,1,0,0,0,1,1,1)^{\mathrm{T}}+Q_{0} Q_{-1} \mathbf{v}_{2}\right), \\
E_{\omega}^{0}\left(N_{-1} \mid X_{T_{1}}=2\right)= & E_{\omega}^{0}\left(N_{-1} \mid A_{1,3}=1\right) \\
= & \mathbf{e}_{3}\left(Q_{0}(1,1,1,0, \ldots, 0)^{\mathrm{T}}+Q_{0} Q_{-1} \mathbf{v}_{2}\right) \\
= & E_{\omega}^{0}\left(N_{-1} \mid A_{1,3}=1\right) \\
= & \mathbf{e}_{3}\left(Q_{0}(1,1,1,0,0,0,1,1,1)^{\mathrm{T}}+Q_{0} Q_{-1} \mathbf{v}_{2}\right) .
\end{aligned}
$$


Substituting (34), (35), and (36) to (31) one concludes that

$$
\begin{aligned}
\frac{\mathrm{dQ}}{\mathrm{d} \mathbf{P}}=2+\sum_{i=0}^{\infty}( & \left.\frac{\alpha_{1+i, 1}}{\alpha_{1+i, 1}+\alpha_{1+i, 2}}, \frac{\alpha_{1+i, 1}}{\alpha_{1+i, 1}+\alpha_{1+i, 2}}, 1,0, \ldots, 0\right) \\
& \times Q_{i} \cdots Q_{0}(1,1,0,1,1,0,1,1,0)^{\mathrm{T}}
\end{aligned}
$$

We mention that the purpose of deriving the invariant measure $Q(\mathrm{~d} \omega)$ and the invariant density $\mathrm{dQ} / \mathrm{d} \mathbf{P}$ is to prove an LLN for the (2-2) RWRE by an approach known as «the environment viewed from particles».

One follows similarly to [8, Corollary 2.1.25] (see also [7]) that $\{\bar{\omega}(n)\}$ is stationary and ergodic under the measure $\bar{Q} \otimes P_{\omega}^{0}$. Define the local drift at site $x$ in the environment $\omega$ as $d(x, \omega)=E_{\omega}^{x}\left(X_{1}-x\right)$. The ergodicity of $\{\bar{\omega}(n)\}$ under $Q \otimes P_{\omega}^{0}$ implies that

$$
\frac{1}{n} \sum_{k=0}^{n-1} d\left(X_{k}, \omega\right)=\frac{1}{n} \sum_{k=0}^{n-1} d(0, \bar{\omega}(k)) \stackrel{n \rightarrow \infty}{\longrightarrow} E_{\bar{Q}}(d(0, \omega)) \quad \bar{Q} \otimes P_{\omega}^{0} \text {-a.s. }
$$

However,

$$
\begin{aligned}
X_{n} & =\sum_{i=1}^{n}\left(X_{i}-X_{i-1}\right)=\sum_{i=1}^{n}\left(X_{i}-X_{i-1}-d\left(X_{i}, \omega\right)\right)+\sum_{i=1}^{n} d\left(X_{i}, \omega\right) \\
& =: M_{n}+\sum_{i=1}^{n} d\left(X_{i}, \omega\right) .
\end{aligned}
$$

Under $P_{\omega}^{0}, M_{n}$ is a martingale, with $\left|M_{n}-M_{n-1}\right| \leqslant 4$ for $\omega \in \Omega$. Hence, with $\mathscr{G}_{n}=\sigma\left(M_{1}, \cdots, M_{n}\right)$,

$$
E_{\omega}^{0}\left(\mathrm{e}^{\lambda M_{n}}\right)=E_{\omega}^{0}\left(\mathrm{e}^{\lambda M_{n-1}} E_{\omega}^{0}\left(\mathrm{e}^{\lambda\left(M_{n}-M_{n-1}\right)} \mid \mathscr{G}_{n}\right)\right) \leqslant E_{\omega}^{0}\left(\mathrm{e}^{\lambda M_{n-1}} \mathrm{e}^{4 \lambda^{2}}\right),
$$

and hence, iterating, $E_{\omega}^{0}\left(\mathrm{e}^{\lambda M_{n}}\right) \leqslant \mathrm{e}^{4 n \lambda^{2}}$ (this is a version of Azuma's inequality, see [2, Corollary 2.4.7]). Then Chebyshev's inequality implies that $P^{0}$-a.s., $\lim _{n \rightarrow \infty} M_{n} / n=0$. Therefore,

$$
\lim _{n \rightarrow \infty} \frac{X_{n}}{n}=E_{\bar{Q}}(d(0, \omega))=: V_{\mathbf{P}}
$$

Further, we calculate $V_{\mathbf{P}}$. Note that

$$
\begin{aligned}
V_{\mathbf{P}} & =E_{\bar{Q}}(d(0, \omega))=E_{\bar{Q}}\left(X_{1}\right) \\
& =E_{\mathbf{P}}\left(\Pi(\omega)\left(2 \omega_{0}(-2)+\omega_{0}(-1)+\omega_{0}(1)+2 \omega_{0}(2)\right)\right) / Q(\Omega) \\
& =\frac{E_{\mathbf{P}}\left(\Pi(\omega)\left(2 \omega_{0}(-2)+\omega_{0}(-1)+\omega_{0}(1)+2 \omega_{0}(2)\right)\right)}{E^{0}\left(T_{1} \mid X_{T_{1}}=1\right)+E^{0}\left(T_{1} \mid X_{T_{1}}=2\right)} .
\end{aligned}
$$


But the denominator in (38) equals

$$
\begin{aligned}
& E_{\mathbf{P}}\left(2+\left(\frac{\alpha_{1,1}}{\alpha_{1,1}+\alpha_{1,2}}, \frac{\alpha_{1,2}}{\alpha_{1,1}+\alpha_{1,2}}, 1,0, \ldots, 0\right)\right. \\
& \left.\quad \times \sum_{i \leqslant 0} Q_{0} \cdots Q_{i}(2,2,1,1,1,0,2,2,1)^{T}\right):=E_{\mathbf{P}}(D(\omega)) .
\end{aligned}
$$

Substituting to (38) one has

$$
V_{\mathbf{P}}=\frac{E_{\mathbf{P}}\left(\Pi(\omega)\left(2 \omega_{0}(-2)+\omega_{0}(-1)+\omega_{0}(1)+2 \omega_{0}(2)\right)\right)}{E_{\mathbf{P}}(D(\omega))} .
$$

Acknowledgements. The authors would like to thank Dr. Hongyan Sun and Lin Zhang for their stimulating discussions. Also we would like to express our gratitude to the referee for his or her careful reading of the first version of paper and useful suggestions for revising the paper.

\section{REFERENCES}

1. Brémont $J$. One-dimensional finite range random walk in random medium and invariant measure equation. - Ann. Inst. H. Poincar Probab. Statist., 2009, v. 45, № 1, p. $70-103$.

2. Dembo A., Zeitouni O. Large Deviations: Techniques and Applications. Berlin: Springer, 1998, $396 \mathrm{p}$.

3. Hong W. M., Wang H. M. Intrinsic branching structure within (L-1) random walk in random environment and its applications. - Infin. Dimens. Anal. Quantum Probab. Relat. Top., 2013, v. 16, № 1.

4. Hong W. M., Zhang L. Branching structure for the transient (1,R)-random walk in random environment and its applications. - Infin. Dimens. Anal. Quantum Probab. Relat. Top., 2010, v. 13, № 4, p. 589-618.

5. Kesten H., Kozlov M.V., Spitzer F. A limit law for random walk in a random environment. - Compositio Math., 1975, v. 30, p. 145-168.

6. Козлов С. М. Метод усреднения и блуждания в однородных средах. - Успехи матем. наук, 1985 , т. 40 , в. 2(242), с. $61-120$.

7. Bolthausen E., Sznitman A.S. Ten lectures on random media. Basel: Birkhäuser Verlag, 2002, $116 \mathrm{p}$.

8. Zeitouni O. Random walks in random environment. - Lecture Notes in Math., 2004, v. 1837, p. $189-312$. 